\title{
Neu bei conrad: CME-Punkte on demand erwerben mit Best-of-Kursen zu Thorax, Herz und Gelenken
}

Seit der Sommerpause des Deutschen Röntgenkongresses finden Sie in unserer Lernplattform conrad Teil 2 des Kurspakets „Best of RÖKO DIGITAL 2020“. Insgesamt können Sie 16 CME-Punkte mit diesen hervorragend bewerteten Vorträgen plus zugehörigen Lernerfolgskontrollen sammeln:

\section{Best of RÖKO DIGITAL 2020 -} Kurspaket Teil 2:

\section{FFF Thorax (4 CME-Punkte)}

S. Dettmer, O. Hamer: FFF Thoraxradiologie: Wissen für die Facharztprüfung

\section{FFF Herzbildgebung (4 CME-Punkte)}

K. Fischbach, S. Reinartz: FFF Herzbildgebung - was muss ich wissen

\section{FFF Muskulo/Gelenke (4 CME-Punkte)}

M. Regier: MRT des Kniegelenks

M. Mack: OSG und Fuß - Was muss ich wissen

\section{Muskuloskelettale Radiologie (4 CME-Punkte)}

M. Regier: Meniskus

M. Mack: Knie: Postoperatives MRT

M. Zanetti: Rotatorenmanschette - was ist befundrelevant und wie beschreibe ich's

T. Diekhoff: Ultraschall kompakt: Hand

Notfall/Intervention II: Ohne den Interventionalisten geht gar nichts (keine CME-Anerkennung)

B. Gebauer: Tiefe Venenthrombose

B. Radeleff: Dringliche Gallenwegsintervention

F. Schaab: Komplikationen nach perkutaner Intervention

Die Kurse sind die Fortsetzung unseres „Best of RÖKO DIGITAL 2020“-Angebots, dessen erstes Paket bereits im Mai veröffentlicht worden ist:

\section{Best of RÖKO DIGITAL 2020 - Kurspaket Teil 1:}

Thoraxradiologie I - Fehlervermeidung (4 CME-Punkte)

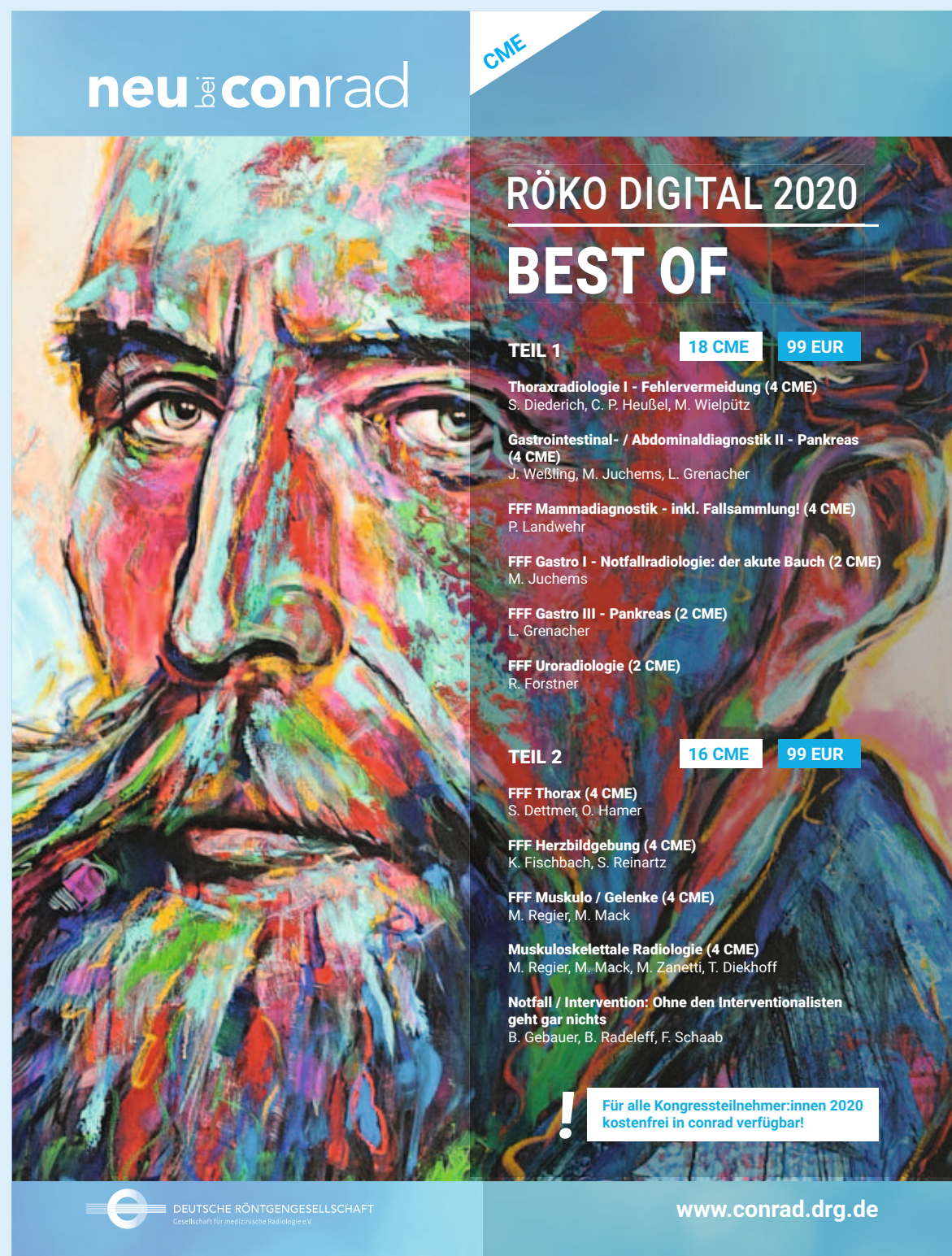

Gastrointestinal-|Abdominaldiagnostik II Pankreas (4 CME-Punkte)

FFF Mammadiagnostik (4 CME-Punkte)

FFF Gastro I - Notfallradiologie: der akute Bauch (2 CME-Punkte)

FFF Gastro III - Pankreas (2 CME-Punkte) FFF Uroradiologie (2 CME-Punkte)

Als Teilnehmer:in des RÖKO DIGITAL 2020 stehen Ihnen die o.g. Kurse automatisch und kostenfrei in conrad zur Verfügung. Für all diejenigen, die kein Kongressticket 2020 erworben haben, ist „Best of RÖKO DIGITAL 2020 - Kurspaket Teil 2“ für 99,00 EUR direkt in conrad buchbar. www.conrad.drg.de

Die Kurse sind verfügbar bis zum 29.05.2022 bzw. 30.06.2022.

conrad - die interaktive Lernplattform der DRG

\section{www.conrad.drg.de}

Kontakt für Rückfragen: DRG-Geschäftsstelle

Anja Johenning

Tel.: 03091607017

Mail: johenning@drg.de 